\title{
Diffusional Kurtosis Imaging of White Matter Degeneration in Glaucoma
}

\author{
Carlo Nucci ${ }^{1, *}$, Francesco Garaci ${ }^{2,3}$, , Simone Altobelli ${ }^{4}$, Francesco Di Ciò ${ }^{4}$, \\ Alessio Martucci ${ }^{1}$ (D), Francesco Aiello ${ }^{1}$, Simona Lanzafame ${ }^{4}$, Francesca Di Giuliano ${ }^{2}$ (D), \\ Eliseo Picchi ${ }^{4,5}$, Silvia Minosse ${ }^{4}$ (D), Massimo Cesareo ${ }^{1}$, Maria Giovanna Guerrisi ${ }^{4}$, \\ Roberto Floris ${ }^{5}$, Luca Passamonti ${ }^{6,7, *(\mathbb{D})}$ and Nicola Toschi ${ }^{4,8}$ (D)
}

1 Ophthalmology Unit, Department of Experimental Medicine, University of Rome Tor Vergata, 00133 Rome, Italy; alessio.martucci@live.it (A.M.); francesco.aiello@ptvonline.it (F.A.); massimo.cesareo@uniroma2.it (M.C.)

2 Neuroradiology Unit, Department of Biomedicine and Prevention, University of Rome Tor Vergata, 00133 Rome, Italy; francescadigiuliano@msn.com

3 San Raffaele Cassino, 03043 Frosinone, Italy

4 Department of Biomedicine and Prevention, University of Rome Tor Vergata, 00133 Rome, Italy; simo85.altobelli@gmail.com (S.A.); francesco2010@hotmail.it (F.D.C.); lanzafame.simona@gmail.com (S.L.); eliseo.picchi@hotmail.it (E.P.); silvia.minosse@libero.it (S.M.); guerrisi@med.uniroma2.it (M.G.G.); toschi@med.uniroma2.it (N.T.)

5 Diagnostic Imaging Unit, Department of Biomedicine and Prevention, University of Rome Tor Vergata, 00133 Rome, Italy; roberto.floris@uniroma2.it

6 Institute of Bioimaging and Molecular Physiology, National Research Council, 20090 Milano, Italy

Department of Clinical Neurosciences, University of Cambridge, Cambridge CB2 0QQ, UK

8 Athinoula A. Martinos Center for Biomedical Imaging and Harvard Medical School, 149 13th Street, Boston, MA 02129, USA

* Correspondence: nucci@med.uniroma2.it (C.N.); francesco.garaci@uniroma2.it (F.G.); lp337@medschl.cam.ac.uk (L.P.);

Tel.: +39-06-7259-6145 (C.N.); +39-06-2090-2471 (F.G.); +44-01223-330293 (L.P.)

Received: 24 July 2020; Accepted: 23 September 2020; Published: 27 September 2020

\begin{abstract}
Glaucoma is an optic neuropathy characterized by death of retinal ganglion cells and loss of their axons, progressively leading to blindness. Recently, glaucoma has been conceptualized as a more diffuse neurodegenerative disorder involving the optic nerve and also the entire brain. Consistently, previous studies have used a variety of magnetic resonance imaging (MRI) techniques and described widespread changes in the grey and white matter of patients. Diffusion kurtosis imaging (DKI) provides additional information as compared with diffusion tensor imaging (DTI), and consistently provides higher sensitivity to early microstructural white matter modification. In this study, we employ DKI to evaluate differences among healthy controls and a mixed population of primary open angle glaucoma patients ranging from stage I to $\mathrm{V}$ according to Hodapp-Parrish-Anderson visual field impairment classification. To this end, a cohort of patients affected by primary open angle glaucoma $(n=23)$ and a group of healthy volunteers $(n=15)$ were prospectively enrolled and underwent an ophthalmological evaluation followed by magnetic resonance imaging (MRI) using a 3T MR scanner. After estimating both DTI indices, whole-brain, voxel-wise statistical comparisons were performed in white matter using Tract-Based Spatial Statistics (TBSS). We found widespread differences in several white matter tracts in patients with glaucoma relative to controls in several metrics (mean kurtosis, kurtosis anisotropy, radial kurtosis, and fractional anisotropy) which involved localization well beyond the visual pathways, and involved cognitive, motor, face recognition, and orientation functions amongst others. Our findings lend further support to a causal brain involvement in glaucoma and offer alternative explanations for a number of multidomain impairments often observed in glaucoma patients.
\end{abstract}


Keywords: primary open angle glaucoma; diffusional kurtosis imaging; diffusion tensor imaging; magnetic resonance imaging

\section{Introduction}

Glaucoma, one of the major leading causes of blindness worldwide [1], is an optic neuropathy characterized by death of the retinal ganglion cells and loss of the axons that make up the optic nerve [2]. These alterations are progressive, and if left untreated can produce visual field deficits and optic nerve head atrophy [3]. Visual impairment due to glaucoma affects daily living with wide-ranging implications in functional domains such as driving, walking, and reading [4-6]. Moreover, several studies have highlighted a possible connection between reductions of the visual field (VF) and a higher likelihood of developing cognitive impairment, both of which are common phenomena in glaucoma patients [7-10]. Interestingly, these observations are in keeping with recent findings supporting the hypothesis that glaucoma could be considered to be a neurodegenerative disorder involving the central nervous system (CNS) [4,11-15]. In this context, several studies have highlighted changes in grey matter (GM) [16,17], functional connectivity [14,18-20], and white matter (WM) $[14,15,20,21]$ in glaucoma patients as compared with controls. For example, Frezzotti et al. [14] found higher axial diffusivity (AD) in the middle cerebellar peduncle, corticospinal tract (CST), anterior thalamic radiation (ATR), and superior longitudinal fascicle (SLF) in primary open angle glaucoma (POAG) patients relative to controls. These results were confirmed in subsequent studies by the same group [20,21]. Furthermore, Boucard et al. found lower fractional anisotropy (FA) in normal tension glaucoma (NTG) patients as compared with healthy controls, in numerous tracts which were part of the primary visual pathway, such as the optic radiation, as well as in tracts located beyond the visual regions, such as the forceps major (FMa), corpus callosum (CC), and the parietal lobe [15].

Diffusion kurtosis imaging (DKI) is a relatively recent diffusion modeling technique which extends diffusion tensor imaging (DTI). DKI allows a more accurate description of the diffusion signal by adding higher order cumulants to the classical diffusion tensor imaging (DTI) model [22-24]. Conceptually, DKI quantifies the deviation from Gaussianity of the self-diffusion probability profile of water molecules in brain tissue. In a complex cytoarchitectonic environment such as white or grey matter, because such deviations are strongly influenced by the underlying microstructure, DKI indices are able to provide complementary information about microstructural alterations as compared with DTI alone [25]. In this context, a small number of previous studies investigated DKI alterations in glaucoma patients using a region-of interest (ROI)-based approach [26,27].

Therefore, the aim of this study was to employ DKI to evaluate normal appearing white matter (NAWM) changes in patients with primary open-angle glaucoma (POAG) as compared with healthy controls in a regionally unbiased, voxel-wise manner. On the basis of prior findings, we hypothesized that group differences would be found both in primary and in secondary tracts. In addition, we posited that differences could also be localized in bundles outside the visual areas, possibly in tracts that interconnect the various lobes, such as the SLF, inferior longitudinal fasciculus (ILF), inferior front-occipital fasciculus (IFOF), and in the uncinate fasciculus (UF).

\section{Methods}

\subsection{Patient Population}

The study's protocol was approved by our Institutional Ethic Committee and adhered to the tenets of the Declaration of Helsinki. All subjects recruited for this cross-sectional study provided written informed consent. The study was based on the retrospective analysis of prospectively gathered data. A total of, 23 POAG patients and 15 age- and gender-matched healthy subjects were recruited from the glaucoma outpatient clinics at the Department of Ophthalmology of our institution. Patients and 
controls were both aged between 50 and 80 years (average age $61.3 \pm 7.9$ ). All 38 subjects enrolled were right-handed (see Table 1). All subjects underwent a complete ophthalmologic evaluation including administration of a medical history questionnaire, with a focus on local and systemic treatments and family history of glaucoma or neurodegenerative disorders. The evaluation included the following: Best Corrected Visual Acuity (BCVA) examination recorded in logMAR using the Early Treatment Diabetic Retinopathy Study chart (Precision Vision, Woodstock, la Salle, PA, USA); slit lamp examination of the anterior segment; intra ocular pressure measurement using Goldmann applanation tonometry; central corneal thickness evaluation using an ultrasound pachymeter (Pachette DGH500, DGH Technology, Inc., Philadelphia, PA, USA); gonioscopy; 24-2 Swedish Interactive Threshold Algorithms Standard Visual Field (VF) testing; and fundus oculi inspection after pupillary dilation. In order to be included, all participants were required to meet the following inclusion criteria: BCVA $>0.3 \log$ MAR, refractive error $< \pm 5$ spherical diopters or $< \pm 3$ cylindrical diopters, transparent ocular media, open anterior chamber (Shaffer classification $>20^{\circ}$ ), and an inter-eye difference not greater than one stage. Exclusion criteria included the following: previous or active optic neuritis, retinal vascular diseases, preproliferative or proliferative diabetic retinopathy, macular degeneration, hereditary retinal dystrophy, use of medication that could affect VF, history of or active neurological, and cerebrovascular or neurodegenerative diseases. The diagnosis of glaucoma was based on the European Glaucoma Society Guidelines.

Table 1. Study population.

\begin{tabular}{ccc}
\hline & POAG & Healthy Controls \\
\hline Population & $n=23$ & $n=15$ \\
Gender & $15 \mathrm{~F}, 8 \mathrm{M}$ & $6 \mathrm{~F}, 9 \mathrm{M}$ \\
$\begin{array}{c}\text { Average age } \\
\text { Stages }\end{array}$ & $61.2 \pm 6.9$ & $60.2 \pm 9.8$ \\
\hline \multicolumn{3}{c}{ Primary Open Angle Glaucoma (POAG). }
\end{tabular}

\subsection{Visual Field Examination and Patient Groups}

VF tests were performed using the Humphrey Visual Field Analyzer with 24-2 Swedish Interactive Threshold Algorithms (Carl Zeiss Meditec Inc., Dublin, CA, USA). Standard Automated Perimetry examinations, as reported in previous studies, were considered to be unreliable and discarded in the presence of fixation losses $>20 \%$ and false-positive and -negative errors $>15 \%$. VF estimates were confirmed in at least 3 subsequent VF examinations and were classified according to the Hodapp-Parrish-Anderson criteria [28]. Patients were divided into stages (I-V) according to visual field impairment. Patients within stage 0 according to the Hodapp-Parrish-Anderson (HAP) classification were excluded from the analysis.

\subsection{Magnetic Resonance Imaging}

Magnetic resonance imaging (MRI) was performed using a 3-Tesla scanner (Achieva 3T Intera, Philips Healthcare, Amsterdam, The Netherlands) equipped with gradients of maximum amplitude and rise time of $80 \mathrm{mT} / \mathrm{m}$ and $200 \mathrm{mT} / \mathrm{m} / \mathrm{ms}$, respectively, and a dedicated 8-channel head coil. The imaging protocol included the following: axial T2-weighted TSE (Turbo Spin Echo) sequence (TR $3000 \mathrm{~ms}$, TE $80 \mathrm{~ms}$, thickness/gap $3 \mathrm{~mm} / 1$ ), axial T2-fluid attenuated inversion recovery (FLAIR) (TR $11000 \mathrm{~ms}$, TE $120 \mathrm{~ms}$, thickness/gap $3 \mathrm{~mm} / 0$ ), sagittal T1-weighted TSE sequence (TR $2000 \mathrm{ms,}$ TE $10 \mathrm{~ms}$, thickness/gap $3 \mathrm{~mm} / 1$ ), and a T1-3D FFE sequence (FOV $224 \times 224$, TR $25 \mathrm{~ms}$ and TE $50 \mathrm{~ms}$ ), which were employed by an expert neuroradiologist for ruling out visible abnormalities. Patients with recognized abnormalities at morphological imaging, such as evidence of cerebrovascular disease on FLAIR sequences, were excluded from the study. Diffusion-weighed imaging was performed using a spin-echo (SE) echo-planar (EPI) single shot sequence with interleaved slice acquisition and the following parameters: TE $89 \mathrm{~ms}$, TR $7774 \mathrm{~ms}$, slice thickness $2.5 \mathrm{~mm}, 60$ slices, no gap, FOV $240 \times 240$, 
matrix $94 \times 94$ voxel, and SENSE reduction factor $\mathrm{R}=2$. Diffusion weighting with two distinct $b$-values (1000 and $2500 \mathrm{~s} / \mathrm{mm}^{2}$ ) was applied in 64 non-coplanar and non-collinear directions. Eight additional non-diffusion-weighted reference images (b0 images) were also acquired.

\subsection{Image Preprocessing and Model Fitting}

Diffusion-weighted images were corrected for subject motion and eddy-current-induced distortions within the ExploreDTI [29] software including geometric image distortion correction and b-matrix reorientation (version 4.8.4 under Matlab 2015, Natick, MA, USA). All DKI metrics were estimated by using the b0 images and complete two-shell data using the DKI model, fitted using constrained nonlinear least squares estimation. From the DKI model, the following parameters were computed: mean kurtosis (MK), kurtosis anisotropy (KA), radial kurtosis (RK), and axial kurtosis (AK). Additionally, we fitted the DTI model to $b=0$ and $b=1000$ data only and extracted the following metrics from the estimated diffusion tensor: mean diffusivity (MD) and fractional anisotropy (FA).

\subsection{Statistical Analysis}

Voxel-wise statistical analysis of all parameters was carried out using Tract Based Spatial Statistics (TBSS) [30] part of FSL [31]. For each map, we tested the null hypothesis of no differences between healthy controls and all patients, as well as the null hypothesis of no association between all brain metrics and disease stage. All tests were performed through separate general linear models (GLMs). The GLMs included age and gender as nuisance covariates as well as full correction for multiple comparisons over space using permutation-based nonparametric inference within the framework of the general linear model (10,000 permutations). The $p$-values were calculated and corrected for multiple comparisons using threshold-free cluster enhancement TFCE [32] employing the two-dimensional (2D) parameter settings, thereby avoiding the use of an arbitrary threshold for the initial cluster-formation. $p$-values $<0.05$ (corrected) was considered to be statistically significant. Anatomical localization of statistically significant clusters according to involved white matter regions and pathways was performed automatically and successively refined manually, in consensus, by two expert neuroradiologists (S.A. and F.G.).

\section{Results}

When comparing all glaucoma patients to healthy controls, we found statistically significant differences in FA (in 1.7\% of voxels included in the TBSS skeleton, Figure 1, Table S1), KA (in 11.4\% of voxels included in the TBSS skeleton, Figure 2, Table S2), MK (in $48.5 \%$ of voxels included in the TBSS skeleton, Figure 3, Table S3) and RK (in 35\% of voxels included in the TBSS skeleton, Figure 4, Table S4). We did not find statistically significant differences in MD and AK metrics. TBSS showed lower FA values in the glaucoma group (GLA) as compared with the controls (CTRL) along and beyond the visual pathway. In particular, we observed lower FA values in several white matter tracts including the genu, the body and the splenium of the CC, in the anterior corona radiata bilateral, in the left superior corona and in the callosal tract (forceps minor, FMi). Statistically significant differences in $\mathrm{KA}$, which again were seen to be lower in GLA as opposed to CTRL, were more diffuse as compared with the differences in FA. They involved the CC (genu, body, and splenium) the posterior thalamic radiation (PTR) bilaterally, including the optic radiation (OR), the left ILF, the left SLF, the left IFOF, in the anterior corona radiata bilaterally, the left superior corona radiata, the left anterior internal capsule, the cingulum and hippocampal part of the cingulate gyrus (CG), the left UF, and the left WM of the temporal and occipital fusiform gyrus. Additionally, in patients with glaucoma, MK values were lower than in the controls in all the same regions where KA differences were observed, with additional and even more widespread differences. In particular, lower values of MK (GLA vs. CTRL) were found in the IFOF bilateral, ILF bilateral, callosal tracts (FMa and FMi), in CST, in the middle cerebellar peduncle, in the anterior and posterior internal capsule and in the WM of the temporal and occipital fusiform gyrus bilateral. Similar results were found for RK. Localizations of white matter clusters in 
Montreal Neurological Institute (MNI) space are reported in Supplementary Materials Tables S1-S4. No significant associations were found between DTI/DKI metrics and the disease stage.

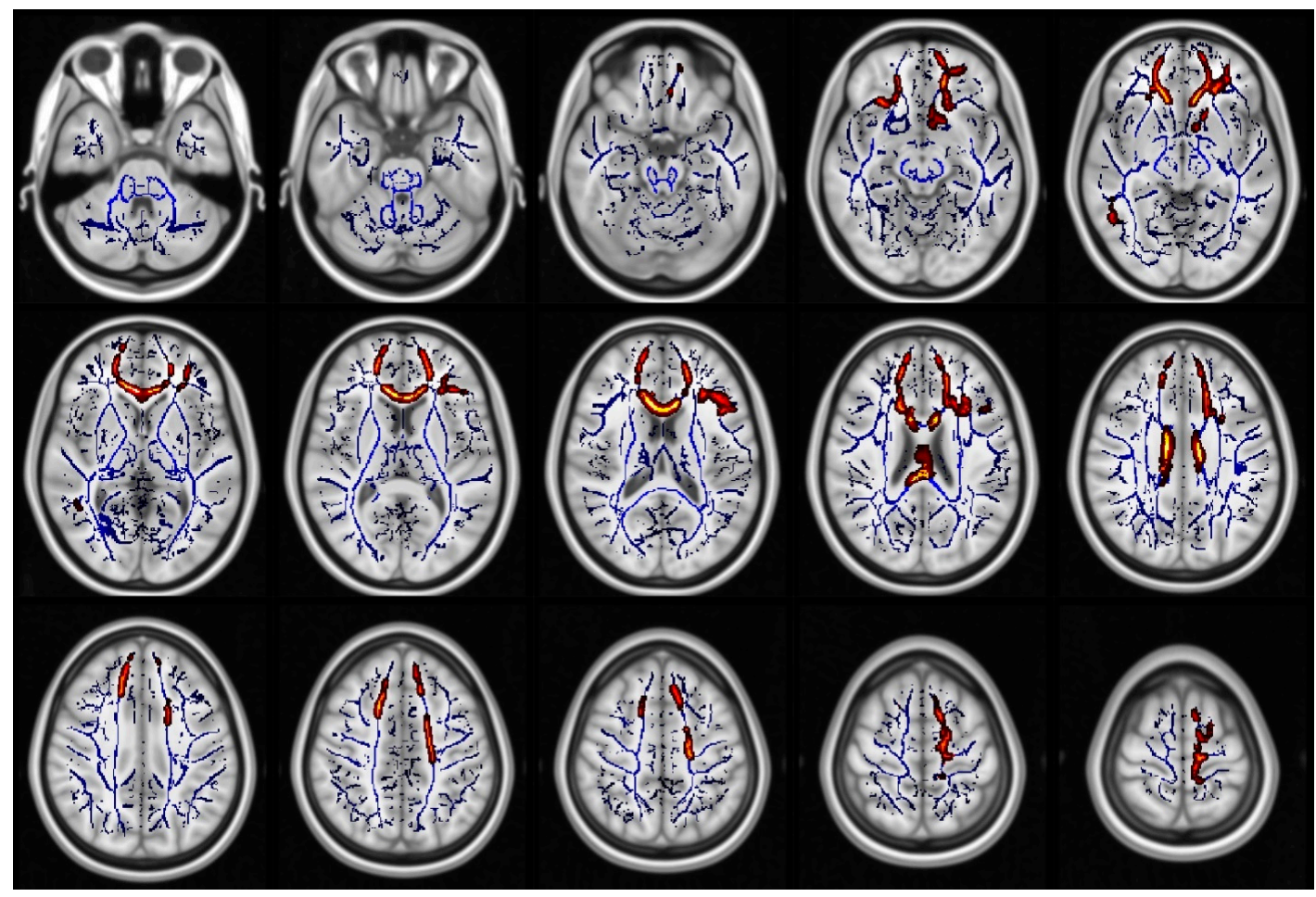

Figure 1. Regions which displayed significant differences in fractional anisotropy (FA) (displayed as red and yellow) (control (CTRL) > glaucoma group (GLA)). The white matter skeleton is shown in blue.

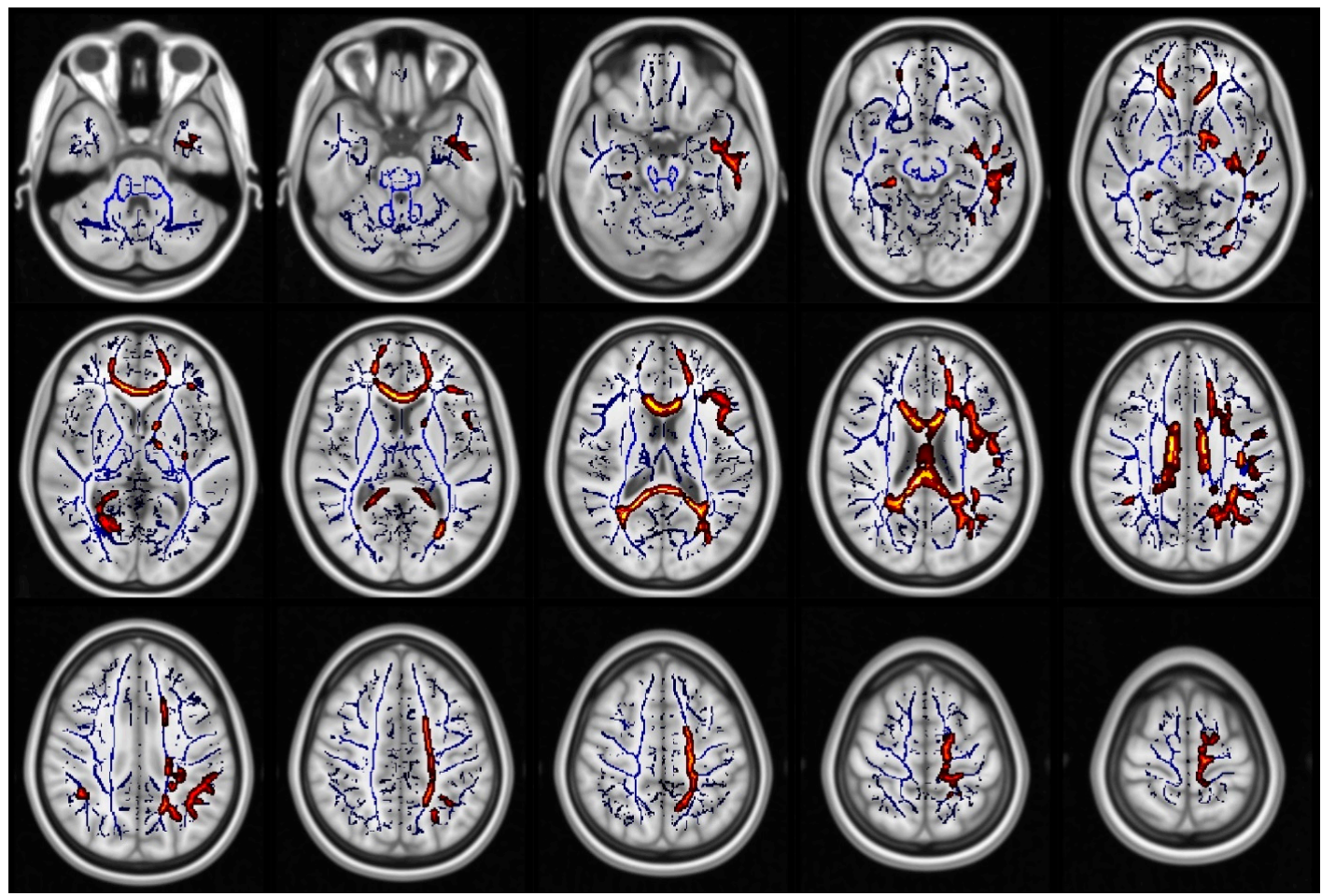

Figure 2. Regions which displayed significant differences in kurtosis anisotropy (KA) (displayed as red and yellow) $($ CTRL) $>$ glaucoma group (GLA)). The white matter skeleton is shown in blue. 


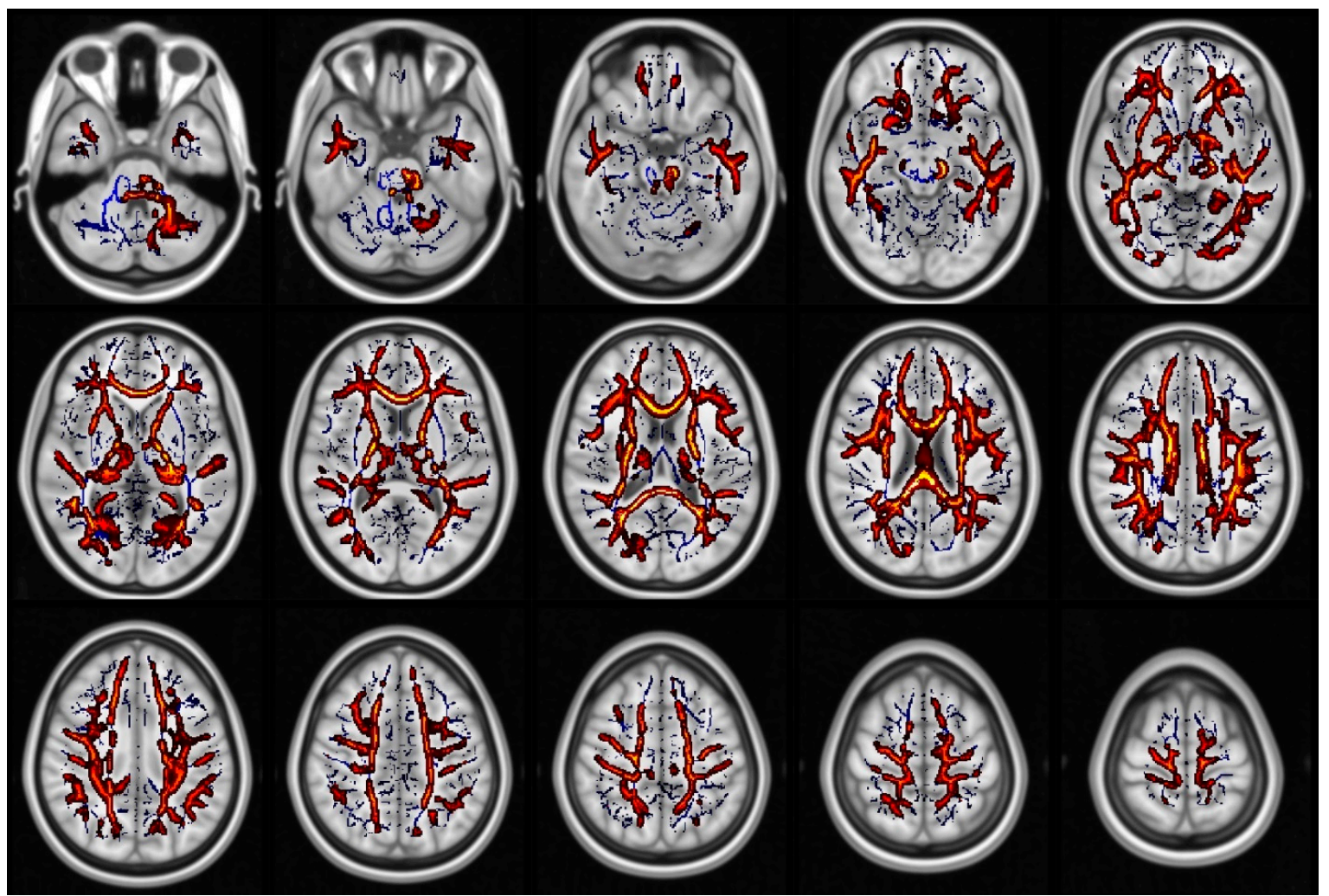

Figure 3. Regions which displayed significant differences in mean kurtosis (MK) (displayed as red and yellow) (CTRL) > glaucoma group (GLA)). The white matter skeleton is shown in blue.

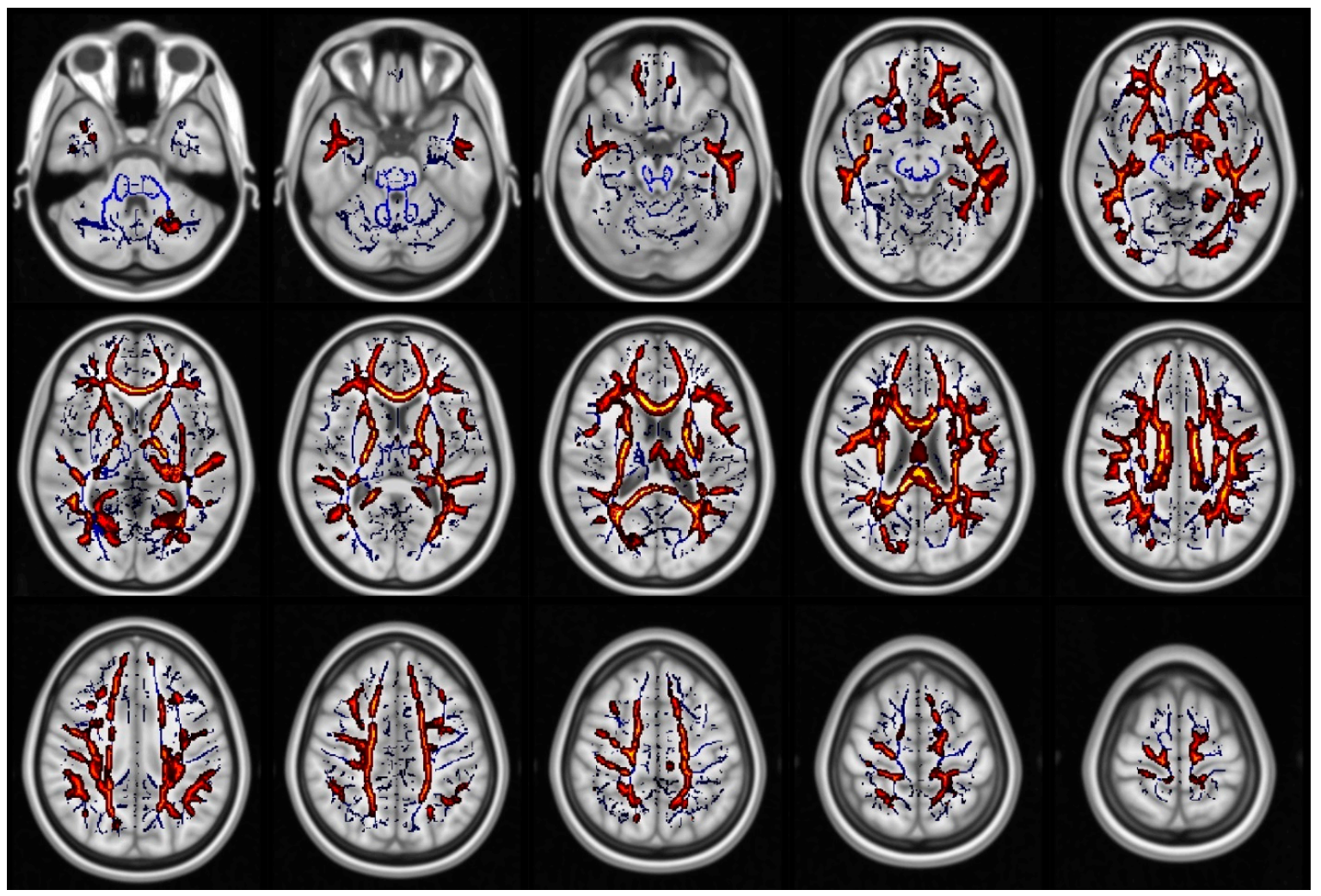

Figure 4. Regions which displayed significant differences in radial kurtosis (RK) (displayed as red and yellow) (CTRL) > glaucoma group (GLA)). The white matter skeleton is shown in blue. 


\section{Discussion}

In this study, we analyzed NAWM in patients with glaucoma using both DTI and DKI, as well as whole-brain TBSS analysis. Although DKI is not yet routinely used in a clinical setting, it has been shown to be extremely effective in some pathologies including cerebrovascular diseases both in animals and humans [33,34], traumatic brain injury [35,36], brain neoplasms [37,38], aging, and neurodegenerative diseases [39,40], inflammatory brain diseases [41] and in other brain and spine pathologies [42,43], suggesting a growing potential for applications both at the level of experimental animal models as well as in human clinical contexts, whenever it is desirable to evaluate early microstructural alterations (e.g., for diagnosis) or to evaluate the response to pharmacological treatment (clinical routine or clinical trials). A recent DKI study [26] demonstrated mean kurtosis (MK) changes in the visual pathway (optic nerves, lateral geniculate nucleus, optic radiations, and visual cortex) of glaucomatous patients. However, the authors did not investigate white matter regions outside the visual pathway, and their study was based on a priori, manual region of interest (ROI) definition. One other study [27] used a ROI-based approach on Broadman areas 17, 18, and 19 in conjunction with DKI indexes on glaucoma patients, which showed bilateral microstructural changes in the aforementioned areas and highlighted a positive correlation between DKI indexes and abnormal effective connectivity in both primary and secondary visual areas and in the parietal lobe, precuneus and angular gyrus, frontal lobe, as well as the superior and middle frontal gyrus. In our study, both DTI and DKI metrics in NAWM were found to be significantly different in glaucoma patients as compared with the controls in widespread regions which were well beyond the visual areas, and the spatial extent of changes in DKI metrics appeared to be much larger that the spatial extent of changes in DTI metrics. As a matter of fact, we found statistically significant differences between GLA and CTRL in only $1.7 \%$ of voxels in FA metrics as compared with the $11.4 \%$ of voxels in the KA metrics. Moreover, MD did not show any statistically significant differences between GLA and CTRL, whereas we found them in $48.5 \%$ of voxels in the MK metric. Although DKI and DTI share the same lack of specificity in terms of disentangling, for example, demyelination, axonal loss, neuroinflammation or edema, this latter finding could be interpreted as a higher sensitivity of DKI vs. DTI in glaucoma.

The differences observed in our study likely represent a loss of interconnection fibers caused by loss and axonal damage comprising the entire visual pathway and the superior cortices involved in the integration of visual information. The alteration of the interconnection fibers (which support visual information processing) may be at the basis of the mismatch between clinical symptoms and visual field defects that are typical of glaucomatous patients and more evident in early disease stages. In this context, the impairment of peripheral vision only partially explains the difficulty these patients have performing actions that require a visual task (both simple and complex). Interestingly, we found higher KA, MK, and RK in healthy controls as compared with POAG in the ILF, which connects the temporal lobe with the occipital lobe [44,45]. This bundle is the anatomical substrate of the ventral stream (VS) [46-48], primarily involved in the recognition of shapes and objects. It originates from the $\mathrm{IV} \alpha$ layer of the visual cortex (V1) and reaches the pole of the temporal lobe, receiving inputs mainly from parvicellular neurons. Lesions of this tract are responsible for symptoms such as prosopagnosia. This is compatible with the statistical differences highlighted in the WM of the fusiform gyrus $[17,49,50]$. The latter is part of the VS and has an important role in object recognition [51], and also in perception and recognition of faces (supported by the so-called fusiform face area) including one's own face [52-55]. Interestingly, patients with glaucoma have an impaired face recognition ability [19,56]. Moreover, the left fusiform gyrus also presents an area called visual word form area (VWFA), which is involved in the recognition of visual words and reading [57-59]. This could offer an alternative explanation for the reading difficulties experienced by glaucoma patients $[5,6,60]$.

The visual pathway has a second system named the dorsal stream (DS), which plays a key role in guiding movement and in visuo-spatial recognition and coordination $[46,48,61,62]$. It originates at the level of the IV $\beta$ layer visual cortex (V1), ends in the posterior parietal lobe, and uses the SLF as anatomical substrate while maintaining deep connections with the VS along its entire course. Injuries 
involving the dorsal stream are responsible for symptoms such as simultanagnosia, visual ataxia, spatial hemineglect, acinetopsia, and apraxia. Interestingly, the DKI differences found in our study were distributed along the course of the anatomical substrates of both neural systems mentioned above, with a specific involvement of the SLF, ILF, and IFO.

Furthermore, and perhaps even more interestingly, we found that patients with glaucoma had diffuse involvement of the white matter tracts in the prefrontal cortex [63], as well as in temporo-parietal regions. Although we did not test for cognitive impairment in our population, previous studies [7-10] have consistently described lower cognitive function in patients with glaucoma which was not merely related to the visual dysfunction per se. The link between glaucoma, cognitive decline, and healthy aging has been recently reported, although a clear pathophysiological framework to explain these associations is still lacking. Our study suggests that the involvement of the non-visual white matter tracts in patients with glaucoma is probably underrecognized and can be mechanistically linked to the increased risk of these patients to express cognitive impairment. Therefore, further research is needed to reveal how the white matter damage may potentially spread from early "bottom-up" visual cortical areas that feed sensory inputs into the brain to more complex, associative, and integrative neural networks that are involved in key cognitive functions such as working memory, attention, and motor control. This has important consequences at the clinical level, for example, to understand a potential risk factor for neurodegenerative disorders such as Alzheimer's disease. For instance, we highlighted lower KA, MK, and RK value in the FM [15]. This tract is an interhemispheric fiber bundle that links the occipital lobes and also continues to the splenium of the CC. The FM is thought to be involved in higher order visual function such as reading, pattern discrimination, perceptual equivalence, and binocular rivalry [64]. Furthermore, pure topographical disorientation has been observed following a lesion in the FM [65], suggesting that the degeneration of this tract could further support the orientation difficulties experienced by glaucoma patients $[5,6,66]$.

Furthermore, we also found lower MK in the CST [14,20]. This tract is primarily involved in motor function, originates in the primary, the premotor, and supplementary, the cortex connects the latter with the motor neurons of the spinal cord [67]. Furthermore, this could lend support to an additional explanation for the motor difficulties that glaucoma patients experience as highlighted in the work of Trivedi et al. [68]. Moreover, we found a decrease in several metrics (FA, KA, MK, and RK) in several parts of the CC, with exception of the rostrum. The CC is a white matter bundle that connects the two hemispheres and is involved in a vast number of functions [69]. Interestingly, there is evidence of secondary WM degeneration in posterior cortical atrophy (PCA), often called the visual variant of Alzheimer's disease [70-72], and CC degeneration has been hypothesized to constitute as a possible characteristic of the PCA [73].

In this context, while the association between glaucoma and risk to develop Alzheimer's disease remains a controversial issue, a more focused approach to study the link between cognitive performance over and above visual deficit (e.g., via auditory-based tasks), glaucoma, and changes in the grey and white matter (via different neuroimaging techniques) have the potential to improve our knowledge regarding the true nature of the associations between glaucoma and $\mathrm{AD}$ that have been reported in past epidemiological research $[4,63,74-76]$.

The Total scan time for the DKI protocol was $9 \mathrm{~min}$ and $20 \mathrm{~s}$. However, recent developments in the realm of multiband echo planar imaging are becoming more and more available in clinical scanners. The application of such techniques can reduce this time to below five minutes with a negligible loss of signal-to-noise ratio $[77,78]$. In this context, the application of DKI in glaucoma, whose social impact is extremely large (more than 60 million patients affected worldwide with a gradual evolution into blindness, i.e., "the silent thief of sight") is significant because the greatest efforts in the clinical field (diagnosis and monitoring of pharmacological response), as well as research, are concentrated in the very early stages of the disease, while neurodegeneration occurs before visual damage can be objectified by the patient. 
Our study is affected by several limitations. We studied a relatively small number of subjects, and therefore our results should be considered to be exploratory. The low sample size is a probable contributor to the null finding when investigating correlation between brain metrics and disease stage directly. To better assess the impact of our findings, a larger sample size and a longitudinal design would be required. Furthermore, the need to employ a larger $b$ value, in the second shell, results in a longer echo time (and subsequently lower signal-to-noise ratio) than what can be achieved in DTI protocols alone. However, it has been shown that even DTI metrics can be estimated with a better accuracy when a DKI term is included [79]. In addition, cognitive testing was not available in our dataset, hence precluding the possibility of formally examining putative associations among glaucoma-related brain changes and cognitive decline.

In conclusion, we have shown widespread normal appearing white matter degeneration in glaucoma which goes well beyond the visual pathways, and whose localization is consistent with a number of multidomain impairments often observed in glaucoma patients. Given that, at present, there are no objective parameters which quantify visual deficit (rather, its estimation relies on a patient's self-rating while performing a visual test), DKI could be a valuable additional toolfor evaluating the clinical course in a disease which does not commonly involve radiological assessment. Since it has been widely demonstrated that neurodegenerative changes in glaucomatous patients are associated with clinical disease severity, from a purely research perspective, our data also suggest a potential application to standardization and quantification in clinical trials.

Supplementary Materials: The following are available online at http://www.mdpi.com/2077-0383/9/10/3122/s1, Table S1: Localization of brain regions which present statistically significant differences in FA (CTRL > GLA). MNI: Montreal Neurological Institute, Table S2: Localizations of brain regions which present statistically significant differences in KA (CTRL > GLA). MNI: Montreal Neurological Institute, Table S3: Localizations of brain regions which present statistically significant differences in MK (CTRL > GLA). MNI: Montreal Neurological Institute, Table S4: Localizations of brain regions which present statistically significant differences in RK (CTRL > GLA). MNI: Montreal Neurological Institute.

Author Contributions: Conceptualization, F.G., C.N., and N.T.; methodology, F.G., C.N., and N.T.; formal analysis, S.L. and N.T.; investigation, A.M., S.L., S.A., F.D.G., and E.P.; data curation, N.T.; writing-original draft preparation, F.G., L.P., F.D.C., and N.T.; writing-review and editing, F.G., L.P., S.A., F.D.C., F.A., F.D.G., E.P., S.M., M.C., M.G.G., R.F., C.N., and N.T.; supervision, F.G., C.N., and N.T. All authors have read and agreed to the published version of the manuscript.

Funding: L.P. is supported by the Medical Research Council (MRC), UK (MR/P01271X/1).

Conflicts of Interest: The authors declare no conflict of interest.

\section{References}

1. Tham, Y.C.; Li, X.; Wong, T.Y.; Quigley, H.A.; Aung, T.; Cheng, C.-Y. Global Prevalence of Glaucoma and Projections of Glaucoma Burden through 2040. Ophthalmology 2014, 121, 2081-2090. [CrossRef] [PubMed]

2. Martucci, A.; Toschi, N.; Cesareo, M.; Giannini, C.; Pocobelli, G.; Garaci, F.; Mancino, R.; Nucci, C. Spectral Domain Optical Coherence Tomography Assessment of Macular and Optic Nerve Alterations in Patients with Glaucoma and Correlation with Visual Field Index. J. Ophthalmol. 2018, 2018, 1-9. [CrossRef] [PubMed]

3. European Glaucoma Society Terminology and Guidelines for Glaucoma, 4th Edition-Chapter 2: Classification and terminologySupported by the EGS Foundation. Br. J. Ophthalmol. 2017, 101, $73-127$. [CrossRef] [PubMed]

4. Nucci, C.; Martucci, A.; Cesareo, M.; Garaci, F.; Morrone, L.A.; Russo, R.; Corasaniti, M.T.; Bagetta, G.; Mancino, R. Links among glaucoma, neurodegenerative, and vascular diseases of the central nervous system. Prog. Brain Res. 2015, 221, 49-65. [PubMed]

5. Sotimehin, A.E.; Ramulu, P.Y. Measuring Disability in Glaucoma. J. Glaucoma 2018, 27, 939-949. [CrossRef] [PubMed]

6. Ramulu, P. Glaucoma and disability: Which tasks are affected, and at what stage of disease? Curr. Opin. Ophthalmol. 2009, 20, 92-98. [CrossRef]

7. Ehrlich, J.R.; Moroi, S.E. Glaucoma, Cognitive Decline, and Healthy Aging. JAMA Ophthalmol. 2017, 135, 740-741. [CrossRef] 
8. Diniz-Filho, A.; Delano-Wood, L.; Daga, F.B.; Cronemberger, S.; Medeiros, F.A. Association between Neurocognitive Decline and Visual Field Variability in Glaucoma. JAMA Ophthalmol. 2017, 135, 734-739. [CrossRef]

9. Honjo, M.; Numaga, J.; Hara, T.; Asaoka, R. The association between structure-function relationships and cognitive impairment in elderly glaucoma patients. Sci. Rep. 2017, 7, 1-9. [CrossRef]

10. Jonas, J.B.; Bin Wei, W.; Zhu, L.P.; Xu, L.; Wang, Y.X. Cognitive Function and Ophthalmological Diseases: The Beijing Eye Study. Sci. Rep. 2018, 8, 4816. [CrossRef]

11. Mancino, R.; Cesareo, M.; Martucci, A.; Di Carlo, E.; Ciuffoletti, E.; Giannini, C.; Morrone, L.A.; Nucci, C.; Garaci, F.; Raffaele, M. Neurodegenerative Process Linking the Eye and the Brain. Curr. Med. Chem. 2019, 26, 3754-3763. [CrossRef] [PubMed]

12. Nucci, C.; Martucci, A.; Cesareo, M.; Mancino, R.; Russo, R.; Bagetta, G.; Cerulli, L.; Garaci, F.G. Brain involvement in glaucoma: Advanced neuroimaging for understanding and monitoring a new target for therapy. Curr. Opin. Pharmacol. 2013, 13, 128-133. [CrossRef] [PubMed]

13. Nucci, C.; Russo, R.; Martucci, A.; Giannini, C.; Garaci, F.; Floris, R.; Bagetta, G.; Morrone, L.A.; Carlo, N.; Rossella, R.; et al. New strategies for neuroprotection in glaucoma, a disease that affects the central nervous system. Eur. J. Pharmacol. 2016, 787, 119-126. [CrossRef] [PubMed]

14. Frezzotti, P.; Giorgio, A.; Motolese, I.; De Leucio, A.; Iester, M.; Motolese, E.; Federico, A.; De Stefano, N. Structural and Functional Brain Changes beyond Visual System in Patients with Advanced Glaucoma. PLoS ONE 2014, 9, e105931. [CrossRef] [PubMed]

15. Boucard, C.C.; Hanekamp, S.; Ćurčić-Blake, B.; Ida, M.; Yoshida, M.; Cornelissen, F.W. Neurodegeneration beyond the primary visual pathways in a population with a high incidence of normal-pressure glaucoma. Ophthalmic Physiol. Opt. 2016, 36, 344-353. [CrossRef]

16. Chen, W.W.; Wang, N.-L.; Cai, S.; Fang, Z.; Yu, M.; Wu, Q.; Tang, L.; Guo, B.; Feng, Y.; Jonas, J.B.; et al. Structural Brain Abnormalities in Patients with Primary Open-Angle Glaucoma: A Study with 3T MR Imaging. Investig. Opthalmol. Vis. Sci. 2013, 54, 545-554. [CrossRef]

17. Wang, Y.; Wang, X.; Zhou, J.; Qiu, J.; Yan, T.; Xie, Y.; Li, L.; Lu, W. Brain morphological alterations of cerebral cortex and subcortical nuclei in high-tension glaucoma brain and its associations with intraocular pressure. Neuroradiology 2019, 62, 495-502. [CrossRef]

18. Giorgio, A.; Zhang, J.; Costantino, F.; De Stefano, N.; Frezzotti, P. Altered Large-Scale Brain Functional Connectivity in Ocular Hypertension. Front. Mol. Neurosci. 2020, 14. [CrossRef]

19. Minosse, S.; Garaci, F.; Martucci, A.; Lanzafame, S.; Di Giuliano, F.; Picchi, E.; Cesareo, M.; Mancino, R.; Guerrisi, M.; Pistolese, C.A.; et al. Primary Open Angle Glaucoma Is Associated with Functional Brain Network Reorganization. Front. Neurol. 2019, 10. [CrossRef]

20. Frezzotti, P.; Giorgio, A.; Toto, F.; De Leucio, A.; De Stefano, N. Early changes of brain connectivity in primary open angle glaucoma. Hum. Brain Mapp. 2016, 37, 4581-4596. [CrossRef]

21. Giorgio, A.; Zhang, J.; Costantino, F.; De Stefano, N.; Frezzotti, P. Diffuse brain damage in normal tension glaucoma. Hum. Brain Mapp. 2017, 39, 532-541. [CrossRef] [PubMed]

22. Jensen, J.H.; Helpern, J.A.; Ramani, A.; Lu, H.; Kaczynski, K. Diffusional kurtosis imaging: The quantification of non-gaussian water diffusion by means of magnetic resonance imaging. Magn. Reson. Med. 2005, 53, 1432-1440. [CrossRef]

23. Jensen, J.H.; Helpern, J.A. MRI quantification of non-Gaussian water diffusion by kurtosis analysis. NMR Biomed. 2010, 23, 698-710. [CrossRef] [PubMed]

24. Fieremans, E.; Jensen, J.H.; Helpern, J.A. White matter characterization with diffusional kurtosis imaging. NeuroImage 2011, 58, 177-188. [CrossRef]

25. Lanzafame, S.; Giannelli, M.; Garaci, F.; Floris, R.; Duggento, A.; Guerrisi, M.; Toschi, N. Differences in Gaussian diffusion tensor imaging and non-Gaussian diffusion kurtosis imaging model-based estimates of diffusion tensor invariants in the human brain. Med. Phys. 2016, 43, 2464-2475. [CrossRef]

26. Xu, Z.; Sun, J.; Zhang, X.; Feng, Y.; Pan, A.; Gao, M.; Zhao, H. Microstructural visual pathway abnormalities in patients with primary glaucoma: $3 \mathrm{~T}$ diffusion kurtosis imaging study. Clin. Radiol. 2018, 73, 591.e9-591.e15. [CrossRef] [PubMed]

27. Li, T.; Qu, X.; Chen, W.; Wang, Q.; Wang, H.; Wang, Y.; Huang, C.; Zhang, X.; Wang, N.; Xian, J. Altered information flow and microstructure abnormalities of visual cortex in normal-tension glaucoma: Evidence from resting-state fMRI and DKI. Brain Res. 2020, 1741, 146874. [CrossRef] 
28. Hodapp, E.; Parrish, R.; Anderson, D.R. Clinical Decisions in Glaucoma; Mosby Incorporated.: St. Louis, MO, USA, 1993.

29. Leemans, A.; Jeurissen, B.; Sijbers, J.; Jones, D.K. ExploreDTI: A graphical toolbox for processing, analyzing, and visualizing diffusion MR data. Int. Soc. Magn. Reson. Med. 2009, 17.

30. Smith, S.M.; Jenkinson, M.; Johansen-Berg, H.; Rueckert, D.; Nichols, T.E.; Mackay, C.; E Watkins, K.; Ciccarelli, O.; Cader, M.Z.; Matthews, P.M.; et al. Tract-based spatial statistics: Voxelwise analysis of multi-subject diffusion data. NeuroImage 2006, 31, 1487-1505. [CrossRef] [PubMed]

31. Jenkinson, M.; Beckmann, C.F.; Behrens, T.E.J.; Woolrich, M.W.; Smith, S.M. Review FSL. NeuroImage 2012, 62, 782-790. [CrossRef]

32. Smith, S.M.; Nichols, T.E. Threshold-free cluster enhancement: Addressing problems of smoothing, threshold dependence and localisation in cluster inference. NeuroImage 2009, 44, 83-98. [CrossRef] [PubMed]

33. Taoka, T.; Fujioka, M.; Kashiwagi, Y.; Obata, A.; Rokugawa, T.; Hori, M.; Masutani, Y.; Aoki, S.; Naganawa, S.; Abe, K. Time Course of Diffusion Kurtosis in Cerebral Infarctions of Transient Middle Cerebral Artery Occlusion Rat Model. J. Stroke Cerebrovasc. Dis. 2016, 25, 610-617. [CrossRef] [PubMed]

34. Weber, R.A.; Hui, E.S.; Jensen, J.H.; Nie, X.; Falangola, M.F.; Helpern, J.A.; Adkins, D.L. Diffusional kurtosis and diffusion tensor imaging reveal different time-sensitive stroke-induced microstructural changes. Stroke 2015, 46, 545-550. [CrossRef] [PubMed]

35. Delouche, A.; Attyé, A.; Heck, O.; Grand, S.; Kastler, A.; Lamalle, L.; Renard, F.; Krainik, A. Diffusion MRI: Pitfalls, literature review and future directions of research in mild traumatic brain injury. Eur. J. Radiol. 2016, 85, 25-30. [CrossRef] [PubMed]

36. Stokum, J.A.; Sours, C.; Zhuo, J.; Kane, R.; Shanmuganathan, K.; Gullapalli, R.P. A longitudinal evaluation of diffusion kurtosis imaging in patients with mild traumatic brain injury. Brain Inj. 2014, 29, 47-57. [CrossRef] [PubMed]

37. Raab, P.; Hattingen, E.; Franz, K.; Zanella, F.E.; Lanfermann, H. Cerebral Gliomas: Diffusional Kurtosis Imaging Analysis of Microstructural Differences. Radiology 2010, 254, 876-881. [CrossRef]

38. Van Cauter, S.; Veraart, J.; Sijbers, J.; Peeters, R.R.; Himmelreich, U.; De Keyzer, F.; Van Gool, S.W.; Van Calenbergh, F.; De Vleeschouwer, S.; Van Hecke, W.; et al. Gliomas: Diffusion Kurtosis MR Imaging in Grading. Radiology 2012, 263, 492-501. [CrossRef]

39. Giannelli, M.; Toschi, N.; Passamonti, L.; Mascalchi, M.; Diciotti, S.; Tessa, C. Diffusion kurtosis and diffusion-tensor MR imaging in Parkinson disease. Radiology 2012, 265, 645-646. [CrossRef]

40. Struyfs, H.; Van Hecke, W.; Veraart, J.; Sijbers, J.; Slaets, S.; De Belder, M.; Wuyts, L.; Peters, B.; Sleegers, K.; Robberecht, C.; et al. Diffusion Kurtosis Imaging: A Possible MRI Biomarker for AD Diagnosis? J. Alzheimer's Dis. 2015, 48, 937-948. [CrossRef]

41. Bester, M.; Jensen, J.H.; Babb, J.; Tabesh, A.; Miles, L.; Herbert, J.; I Grossman, R.; Inglese, M. Non-Gaussian diffusion MRI of gray matter is associated with cognitive impairment in multiple sclerosis. Mult. Scler. J. 2014, 21, 935-944. [CrossRef]

42. Bonilha, L.; Lee, C.Y.; Jensen, J.H.; Tabesh, A.; Spampinato, M.V.; Edwards, J.C.; Breedlove, J.; Helpern, J.A. Altered microstructure in temporal lobe epilepsy: A diffusional kurtosis imaging study. Am. J. Neuroradiol. 2014, 36, 719-724. [CrossRef]

43. Tummala, S.; Palomares, J.; Kang, D.W.; Park, B.; Woo, M.A.; Harper, R.M.; Kumar, R. Global and Regional Brain Non-Gaussian Diffusion Changes in Newly Diagnosed Patients with Obstructive Sleep Apnea. Sleep 2016, 39, 51-57. [CrossRef] [PubMed]

44. Herbet, G.; Zemmoura, I.; Duffau, H. Functional Anatomy of the Inferior Longitudinal Fasciculus: From Historical Reports to Current Hypotheses. Front. Neuroanat. 2018, 12. [CrossRef] [PubMed]

45. Garaci, F.; Picchi, E.; Di Giuliano, F.; Lanzafame, S.; Minosse, S.; Manenti, G.; Pistolese, C.A.; Sarmati, L.; Teti, E.; Andreoni, M.; et al. Cerebral Multishell Diffusion Imaging Parameters are Associated with Blood Biomarkers of Disease Severity in HIV Infection. J. Neuroimaging 2019, 29, 771-778. [CrossRef] [PubMed]

46. Kandel, E.R. Intermediate-level visual processing and visula primitives. In Principles of Neural Science, 5th ed.; McGraw-Hill: New York, NY, USA, 2013.

47. Kandel, E. High-level visual processing: Cognitive influences. In Principles of Neural Science, 5th ed.; McGraw-Hill: New York, NY, USA, 2013.

48. Goodale, M.A.; Milner, A.D. Separate visual pathways for perception and action. Trends Neurosci. 1992, 15, 20-25. [CrossRef] 
49. Jiang, F.; Zeng, F.-F.; Yu, C.; Ye, Y.-Q.; Zeng, X.-J. Altered whole-brain gray matter volume in primary angle closure glaucoma patients. NeuroReport 2018, 29, 1405-1412. [CrossRef]

50. Wang, J.; Li, T.; Wang, N.; Xian, J.; He, H. Graph theoretical analysis reveals the reorganization of the brain network pattern in primary open angle glaucoma patients. Eur. Radiol. 2016, 26, 3957-3967. [CrossRef]

51. Tyler, L.K.; Chiu, S.; Zhuang, J.; Randall, B.; Devereux, B.J.; Wright, P.; Clarke, A.; Taylor, K.I. Objects and categories: Feature statistics and object processing in the ventral stream. J. Cogn. Neurosci. 2013, 25, 1723-1735. [CrossRef]

52. Kanwisher, N.; Yovel, G. The fusiform face area: A cortical region specialized for the perception of faces. Philos. Trans. R. Soc. B Biol. Sci. 2006, 361, 2109-2128. [CrossRef]

53. Iidaka, T. Role of the fusiform gyrus and superior temporal sulcus in face perception and recognition: An empirical review. Jpn. Psychol. Res. 2013, 56, 33-45. [CrossRef]

54. Ma, Y.; Han, S. Functional dissociation of the left and right fusiform gyrus in self-face recognition. Hum. Brain Mapp. 2011, 33, 2255-2267. [CrossRef] [PubMed]

55. Barton, J.J.S.; Press, D.Z.; Keenan, J.P.; O'Connor, M. Lesions of the fusiform face area impair perception of facial configuration in prosopagnosia. Neurology 2002, 58, 71-78. [CrossRef] [PubMed]

56. Glen, F.C.; Crabb, D.P.; Smith, N.D.; Burton, R.; Garway-Heath, D.F. Do patients with glaucoma have difficulty recognizing faces? Investig. Ophthalmol. Vis. Sci. 2012, 53, 3629-3637. [CrossRef] [PubMed]

57. McCandliss, B.D.; Cohen, L.; Dehaene, S. The visual word form area: Expertise for reading in the fusiform gyrus. Trends Cogn. Sci. 2003, 7, 293-299. [CrossRef]

58. Dehaene, S.; Le Clec'H, G.; Poline, J.-B.; Le Bihan, D.; Cohen, L. The visual word form area: A prelexical representation of visual words in the fusiform gyrus. NeuroReport 2002, 13, 321-325. [CrossRef] [PubMed]

59. Dehaene, S.; Cohen, L. The unique role of the visual word form area in reading. Trends Cogn. Sci. 2011, 15, 254-262. [CrossRef]

60. Ramulu, P.Y.; West, S.K.; Muñoz, B.; Jampel, H.D.; Friedman, D.S. Glaucoma and Reading Speed. Arch. Ophthalmol. 2009, 127, 82. [CrossRef]

61. Kandel, E. Visual processing and action. In Principles of Neural Science, 5th ed.; McGraw-Hill: New York, NY, USA, 2013.

62. Gallivan, J.P.; Goodale, M.A. The dorsal “action” pathway. Handb. Clin. Neurol. 2018, 151, 449-466.

63. Murphy, M.C.; Conner, I.P.; Teng, C.Y.; Lawrence, J.D.; Safiullah, Z.; Wang, B.; Bilonick, R.A.; Kim, S.G.; Wollstein, G.; Schuman, J.S.; et al. Retinal Structures and Visual Cortex Activity are Impaired Prior to Clinical Vision Loss in Glaucoma. Sci. Rep. 2016. [CrossRef]

64. Berlucchi, G. Visual interhemispheric communication and callosal connections of the occipital lobes. Cortex 2014, 56, 1-13. [CrossRef]

65. Tamura, I.; Kitagawa, M.; Otsuki, M.; Kikuchi, S.; Tashiro, K.; Dubois, B. Pure Topographical Disorientation Following a Right Forceps Major of the Splenium Lesion: A Case Study. Neurocase 2007, 13, 178-184. [CrossRef] [PubMed]

66. Friedman, D.S.; Freeman, E.; Munoz, B.; Jampel, H.D.; West, S.K. Glaucoma and Mobility Performance. Ophthalmology 2007, 114, 2232-2237.e1. [CrossRef] [PubMed]

67. Welniarz, Q.; Dusart, I.; Roze, E. The corticospinal tract: Evolution, development, and human disorders. Dev. Neurobiol. 2016, 77, 810-829. [CrossRef] [PubMed]

68. Trivedi, V.; Bang, J.W.; Parra, C.; Colbert, M.K.; O'Connell, C.; Arshad, A.; Faiq, M.A.; Conner, I.P.; Redfern, M.S.; Wollstein, G.; et al. Widespread brain reorganization perturbs visuomotor coordination in early glaucoma. Sci. Rep. 2019, 9, 14168. [CrossRef]

69. Filippi, C.G.; Cauley, K. Lesions of the Corpus Callosum and Other Commissural Fibers: Diffusion Tensor Studies. Semin. Ultrasound CT MRI 2014, 35, 445-458. [CrossRef] [PubMed]

70. Levine, D.N.; Lee, J.M.; Fisher, C.M. The visual variant of Alzheimer's disease: A clinicopathologic case study. Neurology 1993, 43, 305. [CrossRef]

71. Bokde, A.L.W.; Pietrini, P.; Ibáñez, V.; Furey, M.L.; Alexander, G.E.; Graff-Radford, N.R.; Rapoport, S.I.; Schapiro, M.B.; Horwitz, B. The effect of brain atrophy on cerebral hypometabolism in the visual variant of Alzheimer disease. Arch. Neurol. 2001, 58, 480-486. [CrossRef]

72. Lee, A.G.; O Martin, C. Neuro-ophthalmic findings in the visual variant of Alzheimer's disease. Ophthalmology 2004, 111, 376-380. [CrossRef] 
73. Yoshida, T.; Shiga, K.; Yoshikawa, K.; Yamada, K.; Nakagawa, M. White Matter Loss in the Splenium of the Corpus callosum in a Case of Posterior Cortical Atrophy: A Diffusion Tensor Imaging Study. Eur. Neurol. 2004, 52, 77-81. [CrossRef]

74. Mancino, R.; Martucci, A.; Cesareo, M.; Giannini, C.; Corasaniti, M.T.; Bagetta, G.; Nucci, C. Glaucoma and Alzheimer Disease: One Age-Related Neurodegenerative Disease of the Brain. Curr. Neuropharmacol. 2018, 16, 971-977. [CrossRef]

75. Inyushin, M.; Zayas-Santiago, A.; Rojas, L.; Kucheryavykh, Y.; Kucheryavykh, L. Platelet-generated amyloid beta peptides in Alzheimer's disease and glaucoma. Histol. Histopathol. 2019, 34, 843-856. [PubMed]

76. Xu, X.; Zou, J.; Geng, W.; Wang, A. Association between glaucoma and the risk of Alzheimer's disease: A systematic review of observational studies. Acta Ophthalmol. 2019, 97, 665-671. [CrossRef]

77. Mitsuda, M.; Suzuki, Y.; Kunimatsu, A.; Kasahara, A.; Watanabe, Y.; Ino, K.; Yano, K.; Ohtomo, K. Feasibility of diffusion tensor imaging at 1.5T using multi-band echo planar acquisition. Magn. Reson. Med. Sci. 2017, 16, 169-175. [CrossRef] [PubMed]

78. Lee, H.-L.; Zhou, X.A.; Li, Z.; Chuang, K.-H. Optimizing diffusion MRI acquisition efficiency of rodent brain using simultaneous multislice EPI. NMR Biomed. 2020. Online ahead of print. [CrossRef] [PubMed]

79. Veraart, J.; Poot, D.; Van Hecke, W.; Blockx, I.; Van Der Linden, A.M.; Verhoye, M.; Sijbers, J. More accurate estimation of diffusion tensor parameters using diffusion kurtosis imaging. Magn. Reson. Med. 2010, 65, 138-145. [CrossRef] [PubMed]

(C) 2020 by the authors. Licensee MDPI, Basel, Switzerland. This article is an open access article distributed under the terms and conditions of the Creative Commons Attribution (CC BY) license (http://creativecommons.org/licenses/by/4.0/). 